\title{
AN ELLIPTIC REGULARITY COEFFICIENT ESTIMATE FOR A PROBLEM ARISING FROM A FREQUENCY DOMAIN TREATMENT OF WAVES
}

\author{
XIAOBING FENG AND DONGWOO SHEEN
}

\begin{abstract}
We consider a sequence of noncoercive elliptic problems, which are the wave equation in the frequency domain, in a rectangular or cubic domain with an absorbing boundary condition. The elliptic regularity coefficient depends on the frequency, and it has a singularity for both zero and infinite frequency. In this paper we derive an elliptic regularity estimate as the frequency tends to zero and infinity.
\end{abstract}

\section{INTRODUCTION}

Consider the scalar wave propagation problem given by

$$
\begin{array}{ll}
u_{t t}-\Delta u=f, & \text { in } \Omega \times[0, \infty), \\
u_{t}+\frac{\partial u}{\partial \nu}=0, & \text { on } \Gamma \times[0, \infty), \\
u=u_{t}=0, & \text { in } \Omega \times\{0\},
\end{array}
$$

where $\Omega=(0,1)^{N}, N=2$ or $3, \Gamma=\partial \Omega$ and $\nu$ is the unit outward normal to $\Gamma$. The boundary condition (1.1.ii) is a standard first-order absorbing boundary condition such that waves arriving normally at the boundary $\Gamma$ are completely annihilated. Instead of solving Problem (1.1) in the space-time domain, we wish to transform the problem into one in the space-frequency domain by taking the Fourier transform in the time variable. Denote the Fourier transform of a function $\rho(t)$ by $\hat{\rho}(\omega)$, where

$$
\hat{\rho}(\omega)=\int_{-\infty}^{\infty} \rho(t) e^{-i \omega t} d t, \quad \rho(t)=\frac{1}{2 \pi} \int_{-\infty}^{\infty} \hat{\rho}(\omega) e^{i \omega t} d \omega .
$$

Thus, in the space-frequency domain, (1.1) is transformed to the family of nonsymmetric, noncoercive, complex-valued elliptic problems of Helmhotz type given by

$$
\begin{aligned}
-\omega^{2} \widehat{u}-\Delta \widehat{u} & =\widehat{f}, & & \text { in } \Omega, \\
\frac{\partial \widehat{u}}{\partial \nu}+i \omega \widehat{u} & =0, & & \text { on } \Gamma,
\end{aligned}
$$

for each $\omega \in \mathbf{R}$.

Received by the editors March 19, 1993.

1991 Mathematics Subject Classification. Primary 35J05, 35J25; Secondary 65 N99.

Key words and phrases. frequency domain, Helmholtz equation, double layer potential. 
One motivation of treating hyperbolic problems like (1.1) in the spacefrequency domain is the effectiveness of parallel computation. Indeed, approximations for $u$ in the space-time domain are obtained sequentially, time step by time step, while it is natural to treat all frequencies for which (1.2) is approximated simultaneously. See [4], [5], [8] for a fuller discussion of parallel algorithms for several wave problems.

Assuming that $f=f(x, t)$ is a real-valued function, we notice that $\widehat{u}(x,-\omega)$ $=\overline{\hat{u}}(x, \omega)$. Therefore, it is sufficient to consider (1.2) for $\omega \geq 0$. For $\omega=0$, (1.2) becomes a Neumann problem and the necessary and sufficient condition for the existence and uniqueness (up to an additive constant) of the solution $\widehat{u}(\cdot, 0)$ is that

$$
\int_{\Omega} \widehat{f}(x, 0) d x=0
$$

(1.3) will be assumed to hold, though the case $\omega=0$ will not be of interest in this paper. For $\omega>0$, there exists a unique solution $\widehat{u}(\cdot, \omega) \in H^{1}(\Omega)$ for a given $\widehat{f}(\cdot, \omega) \in H^{-1}(\Omega)$; see [4]. Denote the solution operator for (1.2) by $T(\omega): L^{2}(\Omega) \rightarrow H^{2}(\Omega)$, so that $u(\cdot, \omega)=T(\omega) f(\cdot, \omega)$. The objective of this paper is to derive precise estimates for elliptic regularity coefficients associated with $T(\omega)$ for $\omega>0$; in particular, the behavior of these bounds as $\omega$ approaches zero and infinity is of critical importance in obtaining an error estimate for a finite element approximation for (1.2) and for inverting back to the time domain by the Fourier inversion formula. These estimates are obtained in the following sections for both two-dimensional and three-dimensional cases. We find bounds for $\widehat{u}, \nabla \widehat{u}$, and $D^{2} \widehat{u}$ that are meromorphic in $\omega$ with a pole of order one at $\omega=0$ and one of order three at $\omega=\infty$. In proving these results we shall use representations in terms of double layer potentials in Lipschitz domains. For the one-dimensional case a complete analysis has been obtained by using Green's function for (1.2); see [5].

\section{Preliminary estimates}

In this section we shall collect some preliminary estimates for the solution of (1.2); these estimates are independent of the dimension of $\Omega$. For notational brevity, replace $\widehat{u}$ and $\widehat{f}$ by $u$ and $f$. Thus, for complex-valued $f \in L^{2}(\Omega)$, we investigate the behavior of the complex-valued solution $u$ of

$$
\begin{aligned}
-\omega^{2} u-\Delta u & =f, & & \text { in } \Omega, \\
\frac{\partial u}{\partial \nu}+i \omega u & =0, & & \text { on } \Gamma,
\end{aligned}
$$

for $\omega>0$, where $\Omega=(0,1)^{N}$ for $N=2$ or $3, \Gamma=\partial \Omega$, and $\nu$ denotes the unit outward normal to $\Gamma$.

Standard notation for function spaces will be used. For example, for $k$ a nonnegative integer and $p \geq 1, W^{k, p}(\Omega)$ will denote the usual complex Sobolev space of $k$ times differentiable functions in the complex Lebesgue space $L^{p}(\Omega)$ and $\|\cdot\|_{W^{k, p}(\Omega)}$ will denote its norm. When $p=2, W^{k, 2}(\Omega)$ will be denoted by $H^{k}(\Omega)$ and $\|\cdot\|_{W^{k, 2}(\Omega)}$ by $\|\cdot\|_{k, \Omega} ;\|\cdot\|_{0, \Omega}$ always means $\|\cdot\|_{L^{2}(\Omega)}$. Let $C^{k, \lambda}(\bar{\Omega})$, for $k$ nonnegative integer and $0<\lambda \leq 1$ denote 
the usual Hölder space of functions whose $k$ th order derivatives are uniformly Hölder continuous with exponent $\lambda$ in $\Omega$, and let $\|\cdot\|_{C^{k, \lambda}(\bar{\Omega})}$ denote its norm. For details of the description of these function spaces, see [1].

In order to get an $H^{2}$-estimate for $u$, we first examine the difference between $\left\|D^{2} u\right\|_{0, \Omega}$ and $\|\Delta u\|_{0, \Omega}$ (see also [7]).

Lemma 2.1. Suppose that $u \in H^{2}(\Omega)$ satisfies the boundary condition (2.1.ii). Then,

$$
\left\|D^{2} u\right\|_{0, \Omega}^{2} \leq\|\Delta u\|_{0, \Omega}^{2} .
$$

Proof. Since the idea of the proof is the same for $N=2$ or 3 , we give the proof only for $N=3$. Before going into the proof, we first introduce some notations. Let

$$
\begin{aligned}
\Gamma & =\Gamma^{x} \cup \Gamma^{y} \cup \Gamma^{z}, \quad \Gamma^{\xi}=\Gamma_{0}^{\xi} \cup \Gamma_{1}^{\xi}, \quad \Gamma_{i}^{\xi}=\{(x, y, z) \in \Gamma \mid \xi=i\}, \\
E^{\xi, \zeta} & =E^{\zeta, \xi}=\bigcup_{i, j=0}^{1} E_{i, j}^{\zeta, \xi}=\bigcup_{i, j=0}^{1} E_{i, j}^{\xi, \zeta}, \\
E_{i, j}^{\xi, \zeta} & =\{(x, y, z) \in \Gamma \mid \xi=i, \zeta=j, \xi \neq \zeta\} \\
{[f]_{E_{i, j}^{\xi, \zeta}} } & =\int_{E_{i, j}^{\xi, \zeta}} f d \gamma, \quad \text { for } \xi, \zeta=x, y, z, \text { and } i, j=0,1 .
\end{aligned}
$$

It is easy to see that the lemma will be proved if we can show the following three identities:

$$
\text { (2.3.i) } \begin{aligned}
\int_{\Omega}\left\{u_{x x} \bar{u}_{y y}+\bar{u}_{x x} u_{y y}\right\} d x d y d z & =2 \operatorname{Re}\left\{\int_{\Omega} u_{x x} \bar{u}_{y y} d x d y d z\right\} \\
& =2\left\|u_{x y}\right\|_{0, \Omega}^{2}+2 \omega^{2}\left[|u|^{2}\right]_{E^{x, y}}, \\
\text { (2.3.ii) } \int_{\Omega}\left\{u_{y y} \bar{u}_{z z}+\bar{u}_{y y} u_{z z}\right\} d x d y d z & =2 \operatorname{Re}\left\{\int_{\Omega} u_{y y} \bar{u}_{z z} d x d y d z\right\} \\
& =2\left\|u_{y z}\right\|_{0, \Omega}^{2}+2 \omega^{2}\left[|u|^{2}\right]_{E^{y, z}}, \\
\text { (2.3.iii) } \int_{\Omega}\left\{u_{z z} \bar{u}_{x x}+\bar{u}_{z z} u_{x x}\right\} d x d y d z & =2 \operatorname{Re}\left\{\int_{\Omega} u_{z z} \bar{u}_{x x} d x d y d z\right\} \\
& =2\left\|u_{z x}\right\|_{0, \Omega}^{2}+2 \omega^{2}\left[|u|^{2}\right]_{E^{z, x}} .
\end{aligned}
$$

Obviously, we need to prove only (2.3.i). It also suffices to assume that $u \in C^{3}(\bar{\Omega}) \cap H^{2}(\Omega)$ and satisfies (2.1.ii). Applying integration by parts and the boundary condition (2.1.ii) repeatedly leads to (2.4)

$$
\begin{aligned}
\int_{\Omega} u_{x x} \bar{u}_{y y} d x d y d z & =-\int_{\Omega} u_{x} \bar{u}_{x y y} d x d y d z+\int_{\Gamma^{x}} \nu_{x} u_{x} \bar{u}_{y y} d \sigma \\
& =\int_{\Omega}\left|u_{x y}\right|^{2} d x d y d z+\int_{\Gamma^{x}} \nu_{x} u_{x} \bar{u}_{y y} d \sigma-\int_{\Gamma^{y}} \nu_{y} u_{x} \bar{u}_{x y} d \sigma \\
& =\left\|u_{x y}\right\|_{0, \Omega}^{2}+\int_{\Gamma^{x}} \frac{\partial u}{\partial \nu} \bar{u}_{y y} d \sigma-\int_{\Gamma^{y}} u_{x}\left(\frac{\partial \bar{u}}{\partial \nu}\right)_{x} d \sigma \\
& =\left\|u_{x y}\right\|_{0, \Omega}^{2}-i \omega \int_{\Gamma^{x}} u \bar{u}_{y y}-i \omega \int_{\Gamma^{y}}\left|u_{x}\right|^{2} d \sigma
\end{aligned}
$$


and

$$
\begin{aligned}
\int_{\Gamma^{x}} u \bar{u}_{y y} d \sigma & =\int_{\Gamma_{0}^{x}} u \bar{u}_{y y} d \sigma+\int_{\Gamma_{1}^{x}} u \bar{u}_{y y} d \sigma \\
& =\int_{\partial \Gamma_{0}^{x}} u \bar{u}_{y} n_{y} d \gamma-\int_{\Gamma_{0}^{x}}\left|u_{y}\right|^{2} d \sigma+\int_{\partial \Gamma_{1}^{x}} u \bar{u}_{y} n_{y} d \gamma-\int_{\Gamma_{1}^{x}}\left|u_{y}\right|^{2} d \sigma \\
& =\int_{E_{0,0}^{x, y} \cup E_{1,0}^{x, y}}\left(-u \bar{u}_{y}\right) d \gamma+\int_{E_{0,1}^{x, y} \cup E_{1,1}^{x, y}} u \bar{u}_{y} d \gamma-\int_{\Gamma^{x}}\left|u_{y}\right|^{2} d \sigma .
\end{aligned}
$$

Since $u$ is sufficiently smooth,

$$
\begin{array}{ll}
\text { on } E_{0,0}^{x, y}: u_{y}=i \omega u, & u_{x}=i \omega u, \\
\text { on } E_{0,1}^{x, y}: u_{y}=-i \omega u, & u_{x}=i \omega u, \\
\text { on } E_{1,0}^{x, y}: u_{y}=i \omega u, & u_{x}=-i \omega u, \\
\text { on } E_{1,1}^{x, y}: u_{y}=-i \omega u, & u_{x}=-i \omega u .
\end{array}
$$

By using these facts, (2.5) then becomes

$$
\begin{aligned}
\int_{\Gamma^{x}} u \bar{u}_{y y} d \sigma= & \int_{E_{0,0}^{x, y} \cup E_{1,0}^{x, y}}(-u) \overline{(i \omega u)} d \gamma+\int_{E_{0,1}^{x, y} \cup E_{1,1}^{x, y}} \overline{(-i \omega u)} d \gamma \\
& -\int_{\Gamma^{x}}\left|u_{y}\right|^{2} d \sigma \\
= & \int_{E^{x, y}} i \omega|u|^{2} d \gamma-\int_{\Gamma^{x}}\left|u_{y}\right|^{2} d \sigma .
\end{aligned}
$$

The combination of (2.4) and (2.6) gives

$$
\begin{aligned}
\int_{\Omega} u_{x x} \bar{u}_{y y} d x d y d z= & \left\|u_{x y}\right\|_{0, \Omega}^{2}+\omega^{2}\left[|u|^{2}\right]_{E^{x, y}}+i \omega \int_{\Gamma^{x}}\left|u_{y}\right|^{2} d \sigma \\
& -i \omega \int_{\Gamma^{y}}\left|u_{x}\right|^{2} d \sigma .
\end{aligned}
$$

Therefore,

$$
\begin{aligned}
\int_{\Omega}\left\{u_{x x} \bar{u}_{y y}+\bar{u}_{x x} u_{y y}\right\} d x d y d z & =2 \operatorname{Re}\left\{\int_{\Omega} u_{x x} \bar{u}_{y y} d x d y d z\right\} \\
& =2\left\|u_{x y}\right\|_{0, \Omega}^{2}+2 \omega^{2}\left[|u|^{2}\right]_{E^{x}, y}
\end{aligned}
$$

for all $u \in C^{3}(\bar{\Omega}) \cap H^{2}(\Omega)$ satisfying (2.1.ii). This completes the proof.

Next, we are going to derive some identities and inequalities satisfied by $u$.

Lemma 2.2. If $u \in H^{2}(\Omega)$ satisfies (2.1), then

$$
\begin{aligned}
-\omega^{2}\|u\|_{0, \Omega}^{2}+\|\nabla u\|_{0, \Omega}^{2} & =\operatorname{Re}\left\{\int_{\Omega} f \bar{u} d x\right\}, \\
\omega|u|_{0, \Gamma}^{2} & =\operatorname{Im}\left\{\int_{\Omega} f \bar{u} d x\right\}, \\
\omega^{2}\|\nabla u\|_{0, \Omega}^{2}-\|\Delta u\|_{0, \Omega}^{2} & =\operatorname{Re}\left\{\int_{\Omega} f \Delta \bar{u} d x\right\}, \\
\omega^{3}|u|_{0, \Gamma}^{2} & =-\operatorname{Im}\left\{\int_{\Omega} f \Delta \bar{u} d x\right\}, \\
\omega^{4}\|u\|_{0, \Omega}^{2}+\|\Delta u\|_{0, \Omega}^{2,} & =2 \omega^{2}\|\nabla u\|_{0, \Omega}^{2}+\|f\|_{0, \Omega}^{2} .
\end{aligned}
$$


Proof. Multiply (2.1.i) by $\bar{u}$ and integrate by parts using (2.1.ii). Then, taking the real and imaginary parts of both sides gives (2.7.i) and (2.7.ii); (2.7.iii) and (2.7.iv) follow in a similar manner if we test (2.1.i) against $\Delta \bar{u}$ instead of $\bar{u}$. Finally, (2.7.v) can be easily obtained from (2.7.i) and (2.7.iii).

Remark. Regarding the regularity assumption in the above lemma we make the following remark. It is proved in [4] that for any $f \in H^{-1}(\Omega)$ the problem (2.1) has a unique slution $u \in H^{1}(\Omega)$. The idea of the proof is to apply the Fredholm Alternative Principle. By using a general regularty result for Laplace equation on convex polygonal domains in [7], we can show that the solution $u$ belongs to $H^{2}(\Omega)$ if the nonhomogeneous term $f$ is in $L^{2}(\Omega)$.

Now, it follows from Lemma 2.2 and Young's inequality that

$$
\begin{aligned}
& \|u\|_{0, \Omega}^{2} \leq \frac{2}{\omega^{2}}\|\nabla u\|_{0, \Omega}^{2}+\frac{1}{\omega^{4}}\|f\|_{0, \Omega}^{2}, \\
& \|\nabla u\|_{0, \Omega}^{2} \leq 2 \omega^{2}\|u\|_{0, \Omega}^{2}+\frac{1}{4 \omega^{2}}\|f\|_{0, \Omega}^{2}, \\
& \|\nabla u\|_{0, \Omega}^{2} \leq \frac{2}{\omega^{2}}\|\Delta u\|_{0, \Omega}^{2}+\frac{1}{4 \omega^{2}}\|f\|_{0, \Omega}^{2}, \\
& \|\Delta u\|_{0, \Omega}^{2} \leq 2 \omega^{2}\|\nabla u\|_{0, \Omega}^{2}+\|f\|_{0, \Omega}^{2} .
\end{aligned}
$$

Using (2.8.ii), (2.8.iv), and (2.2) we can bound $\|u\|_{2, \Omega}^{2}$ as follows:

$$
\begin{aligned}
\|u\|_{2, \Omega}^{2} & =\left\|D^{2} u\right\|_{0, \Omega}^{2}+\|\nabla u\|_{0, \Omega}^{2}+\|u\|_{0, \Omega}^{2} \\
& \leq\|\Delta u\|_{0, \Omega}^{2}+\|\nabla u\|_{0, \Omega}^{2}+\|u\|_{0, \Omega}^{2} \\
& \leq\left(4 \omega^{4}+2 \omega^{2}+1\right)\|u\|_{0, \Omega}^{2}+\left(\frac{1}{4 \omega^{2}}+\frac{3}{2}\right)\|f\|_{0, \Omega}^{2} .
\end{aligned}
$$

Thus, the problem of estimating $u$ in $H^{2}(\Omega)$ is reduced to bounding $\|u\|_{0, \Omega}^{2}$ in terms of $\|f\|_{0, \Omega}^{2}$, and this will be done separately for $N=3$ and $N=2$ in the next two sections.

\section{ESTIMATE FOR $N=3$}

Let $N=3$. An $L^{2}$-estimate for the solution to $(2.1)$ will be established by deriving a representation formula for the solution to (2.1) and using an $L^{2}$ estimate for the double layer potential on Lipschitz domain due to Coifman, McIntosh and Meyer [3]. The fundamental solution (with singularity at $y$ ) to the Helmholtz operator in (2.1.i),

$$
\varphi(x, y)=\frac{1}{4 \pi} \frac{e^{i \omega|x-y|}}{|x-y|},
$$

plays an essential role for the estimate needed in this section.

For sufficiently small $\varepsilon>0$, let $B_{\varepsilon}(y)$ denote the open ball of radius $\varepsilon$ centered at $y, B_{\varepsilon}(y)$ the closure of $B_{\varepsilon}(y)$, and $\Omega_{\varepsilon}=\Omega-\bar{B}_{\varepsilon}(y)$. For each fixed $\omega>0$, a standard argument involving Green's second identity on $\Omega$ leads to Green's representation formula for the solution of (2.1.i): 
Lemma 3.1 (Representation lemma). Let $\varphi(x, y)$ be defined by (3.1) and let $u \in H^{2}(\Omega)$ satisfy (2.1.i). Then the value of $u$ at any point $y \in \Omega$ is given by the formula

$$
u(y)=\int_{\Gamma}\left(\varphi(x, y) \frac{\partial u(x)}{\partial \nu}-u(x) \frac{\partial \varphi(x, y)}{\partial \nu}\right) d \sigma(x)+\int_{\Omega} \varphi(x, y) f(x) d x
$$

Corollary 3.2. Let $u \in H^{2}(\Omega)$ be the solution of (2.1). Then, for any $y \in \Omega$, (3.3)

$$
u(y)=\int_{\Gamma}\left[-i \omega u(x) \varphi(x, y)-u(x) \frac{\partial \varphi(x, y)}{\partial \nu}\right] d \sigma(x)+\int_{\Omega} \varphi(x, y) f(x) d x .
$$
by

For any $y \in \bar{\Omega}$, define a double layer potential with the density $u \in L^{2}(\Gamma)$

$$
I_{1}(y)=\int_{\Gamma} u(x) \frac{\partial}{\partial \nu} \frac{1}{|x-y|} d \sigma(x)=\int_{\Gamma} \frac{u(x)(x-y) \cdot \nu}{|x-y|^{3}} d \sigma(x) .
$$

Here, if $y \in \Gamma$, the integral is understood in the sense of Calderon and Zygmund.

Lemma 3.3. Let $u \in L^{2}(\Gamma)$. Then, there exists a constant $C_{1}$ independent of $u$ such that

$$
\left\|I_{1}\right\|_{0, \Omega}^{2} \leq C_{1}|u|_{0, \Gamma}^{2}
$$

For a proof of Lemma 3.3, we refer to Coifman, McIntosh and Meyer [3].

The main theorem of this section is as follows:

Theorem 3.4. Let $u \in H^{2}(\Omega)$ be the solution of (3.1). Then, there exists a positive constant $C$ such that

$$
\|u\|_{0, \Omega}^{2} \leq C\left[1+\omega^{2}+\alpha(\omega)\right]|u|_{0, \Gamma}^{2}+C\|f\|_{0, \Omega}^{2},
$$

where

$$
\alpha(\omega)=\int_{0}^{\varepsilon_{0}} \frac{\left|1-e^{i \omega r}\right|^{2}}{r^{2}} d r, \quad \varepsilon_{0}>0 .
$$

Proof. We shall estimate each term in the right-hand side of (3.3) in order to obtain the desired estimate (3.5). Observe that, for any $x \in \bar{\Omega}$,

$$
\int_{\Omega} \frac{1}{|x-y|^{2}} d y \leq 4 \pi \int_{0}^{\sqrt{3}} d r=4 \sqrt{3} \pi .
$$

We begin with the last term in the right-hand side of (3.3). By the Hölder inequality and (3.6),

$$
\begin{aligned}
\left|\int_{\Omega} \varphi(x, y) f(x) d x\right|^{2} & \leq \frac{1}{16 \pi^{2}}\left[\int_{\Omega} \frac{|f(x)|}{|x-y|} d x\right]^{2} \\
& \leq \frac{1}{16 \pi^{2}} \int_{\Omega}|f(x)|^{2} d x \int_{\Omega} \frac{1}{|x-y|^{2}} d x \\
& \leq \frac{\sqrt{3}}{4 \pi}\|f\|_{0, \Omega}^{2},
\end{aligned}
$$

so that

$$
\left\|\int_{\Omega} \varphi(x, y) f(x) d x\right\|_{0, \Omega}^{2} \leq \frac{\sqrt{3}}{4 \pi}\|f\|_{0, \Omega}^{2} .
$$


For the first term in the right-hand side of (3.3) we have a similar estimate. By the Hölder inequality, (3.6), and Fubini's theorem,

$$
\begin{aligned}
\left\|\int_{\Gamma}-i \omega u(x) \varphi(x, y) d \sigma(x)\right\|_{0, \Omega}^{2} & \leq \frac{\omega^{2}}{16 \pi^{2}} \int_{\Omega}\left[\int_{\Gamma} \frac{|u(x)|}{|x-y|} d \sigma(x)\right]^{2} d y \\
& \leq \frac{\omega^{2}}{16 \pi^{2}}|u|_{0, \Gamma}^{2} \int_{\Omega} \int_{\Gamma} \frac{1}{|x-y|^{2}} d \sigma(x) d y \\
& =\frac{\omega^{2}}{16 \pi^{2}}|u|_{0, \Gamma}^{2} \int_{\Gamma}\left[\int_{\Omega} \frac{1}{|x-y|^{2}} d y\right] d \sigma(x),
\end{aligned}
$$

which leads to

$$
\left\|\int_{\Gamma}-i \omega u(x) \varphi(x, y) d \sigma(x)\right\|_{0, \Omega}^{2} \leq \frac{3 \sqrt{3}}{2 \pi} \omega^{2}|u|_{0, \Gamma}^{2} .
$$

Finally, let us estimate the second term in the right hand side of (3.3). This term can be rewritten as

$$
\begin{aligned}
\int_{\Gamma} u(x) & \frac{\partial \varphi(x, y)}{\partial \nu} d \sigma(x)=\frac{1}{4 \pi} \int_{\Gamma} u(x) \frac{\partial}{\partial \nu} \frac{e^{i \omega|x-y|}}{|x-y|} d \sigma(x) \\
= & \frac{1}{4 \pi}\left\{\int_{\Gamma} u(x) \frac{\partial}{\partial \nu} \frac{1}{|x-y|} d \sigma(x)+\int_{\Gamma} u(x) \frac{\partial}{\partial \nu} \frac{e^{i \omega|x-y|}-1}{|x-y|} d \sigma(x)\right\} \\
= & \frac{1}{4 \pi}\left\{I_{1}(y)+I_{2}(y)\right\} .
\end{aligned}
$$

Lemma 3.3 applies to the first term $I_{1}$. It remains to obtain an $L^{2}$-estimate of $I_{2}$. It can be decomposed as follows:

$$
I_{2}(y)=\int_{\Gamma} u(x)\left[\left(e^{i \omega|x-y|}-1\right) \frac{(x-y) \cdot \nu}{|x-y|^{3}}+\frac{i \omega e^{i \omega|x-y|}(x-y) \cdot \nu}{|x-y|^{2}}\right] d \sigma(x) .
$$

Therefore,

$$
\begin{aligned}
\left|I_{2}(y)\right| & \leq \int_{\Gamma}|u(x)|\left[\frac{\left|e^{i \omega|x-y|}-1\right|}{|x-y|^{2}}+\frac{\omega}{|x-y|}\right] d \sigma(x) \\
& \leq|u|_{0, \Gamma}\left[\left(\int_{\Gamma} \frac{\left|e^{i \omega|x-y|}-1\right|^{2}}{|x-y|^{4}} d \sigma(x)\right)^{\frac{1}{2}}+\left(\int_{\Gamma} \frac{\omega^{2}}{|x-y|^{2}} d \sigma(x)\right)^{\frac{1}{2}}\right],
\end{aligned}
$$

so that

$$
\left\|I_{2}\right\|_{0, \Omega}^{2} \leq 2|u|_{0, \Gamma}^{2}\left[\int_{\Gamma} \int_{\Omega}\left(\frac{\left|e^{i \omega|x-y|}-1\right|^{2}}{|x-y|^{4}}+\frac{\omega^{2}}{|x-y|^{2}}\right) d y d \sigma(x)\right] .
$$

But, for any $x \in \Gamma$,

$$
\begin{aligned}
\int_{\Omega} \frac{\left|e^{i \omega|x-y|}-1\right|^{2}}{|x-y|^{4}} d y & \leq \int_{B_{\varepsilon_{0}}(x)} \frac{\left|e^{i \omega|x-y|}-1\right|^{2}}{|x-y|^{4}} d y+\int_{\Omega_{\varepsilon_{0}}} \frac{4}{|x-y|^{4}} d y \\
& \leq \frac{16 \pi}{\varepsilon_{0}}+4 \pi \int_{0}^{\varepsilon_{0}} \frac{\left|1-e^{i \omega r}\right|^{2}}{r^{2}} d r
\end{aligned}
$$


from which we obtain, again using (3.6), the inequality (3.10)

$$
\begin{aligned}
\left\|I_{2}\right\|_{0, \Omega}^{2} & \leq 2|u|_{0, \Gamma}^{2}\left\{\int_{\Gamma}\left[\frac{16 \pi}{\varepsilon_{0}}+4 \pi \int_{0}^{\varepsilon_{0}} \frac{\left|1-e^{i \omega r}\right|^{2}}{r^{2}} d r+4 \sqrt{3} \pi \omega^{2}\right] d \sigma(x)\right\} \\
& \leq 12\left[\frac{16 \pi}{\varepsilon_{0}}+4 \pi \alpha(\omega)+4 \sqrt{3} \pi \omega^{2}\right]|u|_{0, \Gamma}^{2},
\end{aligned}
$$

where

$$
\alpha(\omega)=\int_{0}^{\varepsilon_{0}} \frac{\left|1-e^{i \omega r}\right|^{2}}{r^{2}} d r .
$$

Now, combining (3.3)-(3.4) and (3.7)-(3.10) gives

$$
\|u\|_{0, \Omega}^{2} \leq C\left\{1+\omega^{2}+\alpha(\omega)\right\}|u|_{0, \Gamma}^{2}+C\|f\|_{0, \Omega}^{2}
$$

for a computable constant $C=C\left(\varepsilon_{0}\right)$ which is independent of $u$ and $\omega$. The proof, therefore, is complete.

Lemma 3.5. For $\omega>0$,

$$
\alpha(\omega) \leq \begin{cases}2 \omega^{2}, & \text { for all } \omega>0, \\ 2 \omega, & \text { for } \omega \geq 1 .\end{cases}
$$

Proof. Let $\varepsilon_{0}=2$. Then,

$$
\alpha(\omega)=2 \int_{0}^{2} \frac{1-\cos \omega r}{r^{2}} d r=2 \omega \int_{0}^{\omega} \frac{\sin ^{2} t}{t^{2}} d t .
$$

The integrand can be bounded for all $t$ by 1 and by $t^{-2}$ for $t \geq 1$, and the lemma follows immediately.

We now come to our goal of establishing elliptic regularity coefficient estimates for the solution of $(2.1)$.

Theorem 3.6. Let $u \in H^{2}(\Omega)$ be the solution of (3.1) for $\omega>0$. Then there exist positive constants $a_{j}, j=0,1,2,3$, such that

$$
\begin{aligned}
\|u\|_{0, \Omega} & \leq a_{0}\left(\omega+\omega^{-1}\right)\|f\|_{0, \Omega}, \\
\|\nabla u\|_{0, \Omega} & \leq a_{1}\left(\omega^{2}+\omega^{-1}\right)\|f\|_{0, \Omega}, \\
\left\|D^{2} u\right\|_{0, \Omega}^{2} & \leq a_{2}\left(\omega^{3}+1\right)\|f\|_{0, \Omega}, \\
\|u\|_{2, \Omega} & \leq a_{3}\left(\omega^{3}+\omega^{-1}\right)\|f\|_{0, \Omega},
\end{aligned}
$$

Proof. By (2.7.ii),

$$
\begin{aligned}
|u|_{0, \Gamma}^{2} & \leq \frac{1}{\omega}\|f\|_{0, \Omega}\|u\|_{0, \Omega} \\
& \leq \begin{cases}\varepsilon\|u\|_{0, \Omega}^{2}+\frac{1}{4 \varepsilon \omega^{2}}\|f\|_{0, \Omega}^{2}, & \text { if } \omega \leq 1, \\
\frac{\varepsilon}{\omega^{2}}\|u\|_{0, \Omega}^{2}+\frac{1}{4 \varepsilon}\|f\|_{0, \Omega}^{2}, & \text { if } \omega>1 .\end{cases}
\end{aligned}
$$

It follows from (3.5), (3.11), and (3.13) that

$$
\|u\|_{0, \Omega}^{2} \leq C\|f\|_{0, \Omega}^{2}+ \begin{cases}C\left(1+\omega^{2}\right)\left(\varepsilon\|u\|_{0, \Omega}^{2}+\frac{1}{4 \varepsilon \omega^{2}}\|f\|_{0, \Omega}^{2}\right), & \text { if } \omega \leq 1, \\ C\left(1+\omega^{2}\right)\left(\frac{\varepsilon}{\omega^{2}}\|u\|_{0, \Omega}^{2}+\frac{1}{4 \varepsilon}\|f\|_{0, \Omega}^{2}\right), & \text { if } \omega>1 .\end{cases}
$$


Choose $\varepsilon$ small enough so that there exist some positive constant $a_{0}^{2}$ such that

Therefore,

$$
\|u\|_{0, \Omega}^{2} \leq \begin{cases}\frac{a_{0}^{2}}{\omega^{2}}\|f\|_{0, \Omega}^{2}, & \text { if } \omega \leq 1 \\ a_{0}^{2} \omega^{2}\|f\|_{0, \Omega}^{2}, & \text { if } \omega>1\end{cases}
$$

$$
\|u\|_{0, \Omega} \leq a_{0}\left(\omega+\omega^{-1}\right)\|f\|_{0, \Omega} .
$$

This is exactly (3.12.i). (3.12.ii), (3.12.iii) and (3.12.iv) are the immediate consequences of (3.13.i), (2.8.ii), (2.8iv), (2.2) and (2.9). The proof, therefore, is complete.

\section{ESTIMATE FOR $N=2$}

In this section we present an analogous study of elliptic regularity for solutions of (2.1) in a square domain in $\mathbf{R}^{2}$. The same method as used in previous section will be employed, but in the two-dimensional case the fundamental solution for the Helmhotz operator, which is given by the Hankel function, has a nonelementary form, this will leads to a more complicated and delicate analysis. Nevertheless, the same estimate is obtained as the one in the three-dimensional case.

The fundamental solution $\psi(x, y)$ to the Helmhotz equation (2.1.i) with singularity at $y$ in the two-dimensional case is given by

$$
\psi(x, y)=\frac{i}{4 \pi} H_{0}^{(1)}(\omega|x-y|),
$$

where $H_{0}^{(1)}(r)$ denotes the Hankel function of the first kind of order zero. More about the Hankel functions, we refer to [9] and [11]. For this Hankel function there are the following well-known asymptotic expansion results.

Lemma 4.1. For the Hankel function of the first kind of order zero $H_{0}^{(1)}(r)$ there holds the following small and large asymptotic expansion formulas: For some sufficiently small positive number $r_{0}$, if $r<r_{0}$, then

$$
\begin{aligned}
H_{0}^{(1)}(r) & =1+2\left(\gamma+\ln \frac{r}{2}\right) i+O\left(r^{2}\right), \\
\frac{d H_{0}^{(1)}(r)}{d r} & =-\left(\frac{r}{2}-\frac{2}{r} i\right)+O\left(r \ln \frac{r}{2}\right) ;
\end{aligned}
$$

and for some sufficiently large positive number $r_{1}$, if $r>r_{1}$, then

$$
\begin{aligned}
H_{0}^{(1)}(r) & =\sqrt{\frac{2}{\pi r}} e^{i\left(r-\frac{\pi}{4}\right)}\left(1+O\left(\frac{1}{r}\right)\right), \\
\frac{d H_{0}^{(1)}(r)}{d r} & =-\sqrt{\frac{2}{\pi r}} e^{i\left(r-\frac{3 \pi}{4}\right)}\left(1+O\left(\frac{1}{r}\right)\right),
\end{aligned}
$$

where $\gamma$ is some constant.

For the derivation of these asymptotic expansion formulas, see [9] and [11].

Similar to the three-dimensional case, there holds the following representation lemma. 
Lemma 4.2 (Representation lemma). Let $u \in H^{2}(\Omega)$ satisfy (2.1.i). Then, for any $y \in \Omega$,

$$
u(y)=\int_{\Gamma}\left[\psi(x, y) \frac{\partial u(x)}{\partial \nu}-u(x) \frac{\partial \psi(x, y)}{\partial \nu}\right] d \sigma(x)+\int_{\Omega} \psi(x, y) f(x) d x .
$$

Corollary 4.3. Let $u \in H^{2}(\Omega)$ be the solution of (2.1). Then, for any $y \in \Omega$,

$$
u(y)=\int_{\Gamma}\left[-i \omega u(x) \psi(x, y)-u(x) \frac{\partial \psi(x, y)}{\partial \nu}\right] d \sigma(x)+\int_{\Omega} \psi(x, y) f(x) d x .
$$

For any $y \in \bar{\Omega}$, a double layer potential with the density $u \in L^{2}(\Gamma)$ is then defined by

$$
J_{1}(y)=\frac{1}{2 \pi} \int_{\Gamma} u(x) \frac{\partial}{\partial \nu} \ln \frac{1}{|x-y|} d \sigma(x)=-\frac{1}{2 \pi} \int_{\Gamma} \frac{u(x)(x-y) \cdot \nu}{|x-y|^{2}} d \sigma(x) .
$$

If $y \in \Gamma$, the integral is again understood in the sense of Calderón and Zygmund.

Lemma 4.4. Let $y \in \bar{\Omega}$. Then there exists $C>0$, independent of $u \in L^{2}(\Gamma)$, such that

$$
\left\|J_{1}\right\|_{0, \Omega}^{2} \leq C|u|_{0, \Gamma}
$$

For a proof of Lemma 4.4, we again refer to [3].

We now are ready to state the the following main theorem of this section.

Theorem 4.5. Let $u \in H^{2}(\Omega)$ be the solution of (2.1). Then there exists a positive constant $C$ such that

$\|u\|_{0, \Omega}^{2} \leq \begin{cases}C\left(1+\omega^{2}|\ln \omega|^{2}\right)|u|_{0, \Gamma}^{2}+C\left(1+|\ln \omega|^{2}\right)\|f\|_{0, \Omega}^{2}, & \text { if } \omega<r_{0} / \sqrt{2}, \\ C\left(1+\omega^{2}\right)|u|_{0, \Gamma}^{2}+C\|f\|_{0, \Omega}^{2}, & \text { if } \omega \geq r_{0} / \sqrt{2} .\end{cases}$

Proof. We first notice that the Hankel function $H_{0}^{(1)}(r)$ and its first order derivative are continuous everywhere except at $r=0$. This simple fact and (4.2.iii)-(4.2.iv) then imply that for any $0<\varepsilon_{0}<\infty$ there is some positive constant $C_{0}$, which depends only on $\varepsilon_{0}$ and $r_{1}$, such that

$$
\begin{aligned}
&|\psi(x, y)| \leq C_{0}, \quad \text { for all } \varepsilon_{0} \leq \omega|x-y|, \\
&\left|\frac{\partial \psi(x, y)}{\partial x_{k}}\right| \leq \omega C_{0}, \quad \text { for all } \varepsilon_{0} \leq \omega|x-y|<\infty .
\end{aligned}
$$

Let

$$
A_{s, t}(y)=\{x \in \Omega|s<\omega| x-y \mid<t\}, \quad \forall 0 \leq s<t<\infty
$$

for any fixed $\omega>0$ and $\bar{A}_{s, t}(y)$ be the closure of $A_{s, t}(y)$ in $\Omega$. Note that $\left|A_{0, \varepsilon_{0}}(y)\right|=\pi \varepsilon_{0}^{2} / \omega^{2}$.

By (4.1.i), (4.2.ii) and (4.6) we have for any $0<\omega<r_{0} / \sqrt{2}$

$$
\begin{aligned}
\int_{\Omega}|\psi(x, y)|^{2} d x & =\frac{1}{(4 \pi)^{2}} \int_{\Omega}\left|i-2\left(\gamma+\ln \frac{\omega|x-y|}{2}\right)+O\left(\omega^{2}|x-y|^{2}\right)\right|^{2} d x \\
& \leq C_{1}\left(r_{0}\right) \int_{\Omega}\left[1+|\ln \omega|^{2}+|\ln | x-\left.y\right|^{2}\right] d x \\
& \leq C_{2}\left(r_{0}\right)\left(1+|\ln \omega|^{2}\right),
\end{aligned}
$$


and for all $\omega \geq r_{0} / \sqrt{2}$

(4.7.ii)

$$
\begin{aligned}
\int_{\Omega}|\psi(x, y)|^{2} d x & =\int_{A_{0, r_{0}}(y)}|\psi(x, y)|^{2} d x+\int_{A_{r_{0}, \infty}(y)}|\psi(x, y)|^{2} d x \\
& \leq C_{3}\left(r_{0}\right) \int_{A_{0, r_{0}(}(y)}\left[1+|\ln \omega|^{2}+|\ln | x-y||^{2}\right] d x+C_{0}^{2}\left(r_{0}, r_{1}\right) \\
& \leq C_{4}\left(r_{0}\right)\left[\frac{1+|\ln \omega|}{\omega}\right]^{2}+C_{0}^{2}\left(r_{0}, r_{1}\right) \\
& \leq C_{5}\left(r_{0}\right)\left[\frac{\sqrt{2}}{r_{0}}+\frac{\left|\ln r_{0}\right|}{r_{0}}+\frac{1}{e}\right]^{2}+C_{0}^{2}\left(r_{0}, r_{1}\right) \\
& =C_{6}\left(r_{0}, r_{1}\right) .
\end{aligned}
$$

Similarly, for any $\omega>0$ there holds

(4.8)

$$
\begin{aligned}
\int_{\Omega} \mid & \frac{\partial \psi(x, y)}{\partial x_{k}}-\left.\frac{1}{2 \pi} \frac{\partial}{\partial x_{k}} \ln \frac{1}{|x-y|}\right|^{2} d x \\
= & \int_{A_{0, r_{0}(y)}}\left|\frac{\partial \psi(x, y)}{\partial x_{k}}-\frac{1}{2 \pi} \frac{\partial}{\partial x_{k}} \ln \frac{1}{|x-y|}\right|^{2} d x \\
& +\int_{A_{r_{0}, \infty}(y)}\left|\frac{\partial \psi(x, y)}{\partial x_{k}}-\frac{1}{2 \pi} \frac{\partial}{\partial x_{k}} \ln \frac{1}{|x-y|}\right|^{2} d x \\
\leq & \int_{A_{0, r_{0}}(y)}\left|\omega\left[\frac{-\omega|x-y|}{8 \pi}+O\left(\omega|x-y| \ln \frac{\omega|x-y|}{2}\right)\right] \cdot \frac{x_{k}-y_{k}}{\left|x_{k}-y_{k}\right|}\right|^{2} d x \\
& +\int_{A_{r_{0}, \infty}(y)} 2\left[\left|\frac{\partial \psi(x, y)}{\partial x_{k}}\right|^{2}+\omega^{2}\left|\frac{1}{2 \pi|x-y| \omega} \cdot \frac{x_{k}-y_{k}}{\left|x_{k}-y_{k}\right|}\right|^{2}\right] d x \\
\leq & C_{7}\left(r_{0}\right)\left(\omega^{2}+\omega^{4}\right)\left(\frac{r_{0}}{\omega}\right)^{2}+C_{8}\left(r_{0}\right) \omega^{2} \\
\leq & C_{9}\left(r_{0}\right)\left(1+\omega^{2}\right) .
\end{aligned}
$$

Like in the previous section we shall estimate each term in the right-hand side of $(4.3)$ in order to obtain the desired estimate (4.5). We begin with the last term in the right-hand side of (4.3). By the Hölder inequality and (4.7)

$$
\begin{aligned}
\int_{\Omega}\left|\int_{\Omega} \psi(x, y) f(x) d x\right|^{2} d y & \leq\|f\|_{0, \Omega}^{2} \int_{\Omega} \int_{\Omega}|\psi(x, y)|^{2} d x d y \\
& \leq \begin{cases}C_{2}\left(1+|\ln \omega|^{2}\right)\|f\|_{0, \Omega}^{2}, & \text { if } \omega<r_{0} / \sqrt{2}, \\
C_{6}\|f\|_{0, \Omega}^{2}, & \text { if } \omega \geq r_{0} / \sqrt{2} .\end{cases}
\end{aligned}
$$

The first term in the right-hand side of (4.3) can be treated as in the threedimensional case. That is, by the Hölder inequality, Fubini's theorem and (4.7) 
$(4.10)$

$$
\begin{aligned}
& \left\|\int_{\Gamma}-i \omega u(x) \psi(x, y) d \sigma(x)\right\|_{0, \Omega}^{2} \leq \omega^{2} \int_{\Omega}\left|\int_{\Gamma} u(x) \psi(x, y) d \sigma(x)\right|^{2} d y \\
& \quad \leq \omega^{2}|u|_{0, \Gamma}^{2} \int_{\Omega} \int_{\Gamma}|\psi(x, y)|^{2} d \sigma(x) d y \\
& \quad=\omega^{2}|u|_{0, \Gamma}^{2} \int_{\Gamma}\left[\int_{\Omega}|\psi(x, y)|^{2} d y\right] d \sigma(x) \\
& \quad \leq \begin{cases}4 C_{2} \omega^{2}\left(1+|\ln \omega|^{2}\right)|u|_{0, \Gamma}^{2}, & \text { if } \omega<r_{0} / \sqrt{2}, \\
4 C_{6} \omega^{2}|u|_{0, \Gamma}^{2}, & \text { if } \omega \geq r_{0} / \sqrt{2} .\end{cases}
\end{aligned}
$$

Finally, for the second term in the right-hand side of (4.3) we rewrite it as following

$$
\begin{aligned}
\int_{\Gamma} u(x) \frac{\partial \psi(x, y)}{\partial \nu} d \sigma(x)= & \frac{1}{2 \pi} \int_{\Gamma} u(x) \ln \frac{1}{|x-y|} d \sigma(x) \\
& +\int_{\Gamma} u(x) \frac{\partial}{\partial \nu}\left[\psi(x, y)-\frac{1}{2 \pi} \ln \frac{1}{|x-y|}\right] d \sigma(x) \\
= & J_{1}(y)+J_{2}(y) .
\end{aligned}
$$

By Lemma 4.4 $\left\|J_{1}\right\|_{0, \Omega}^{2} \leq C|u|_{0, \Gamma}^{2}$, and by (4.8)

$$
\left\|J_{2}\right\|_{0, \Omega}^{2} \leq 8 C_{9}\left(1+\omega^{2}\right)|u|_{0, \Gamma}^{2} \text {. }
$$

So

$$
\left\|\int_{\Gamma} u(x) \frac{\partial \psi(x, y)}{\partial \nu} d \sigma(x)\right\|_{0, \Omega}^{2} \leq C_{10}\left(1+\omega^{2}\right)|u|_{0, \Gamma}^{2} .
$$

The proof of the theorem is complete after combining (4.9), (4.10), (4.11).

We shall conclude this section by stating the following elliptic regularity estimate. Since the idea of the proof is exactly the same as one for Theorem 3.6, we shall only give a brief sketch of the proof.

Theorem 4.7. Let $u \in H^{2}(\Omega)$ be the solution of (4.1) for $\omega>0$. There exist positive constants $b_{j}, j=0,1,2,3$, such that

$$
\begin{aligned}
\|u\|_{0, \Omega} & \leq b_{0}\left(\omega+\omega^{-1}\right)\|f\|_{0, \Omega}, \\
\|\nabla u\|_{0, \Omega} & \leq b_{1}\left(\omega^{2}+\omega^{-1}\right)\|f\|_{0, \Omega}, \\
\left\|D^{2} u\right\|_{0, \Omega} & \leq b_{2}\left(\omega^{3}+1\right)\|f\|_{0, \Omega}, \\
\|u\|_{2, \Omega} & \leq b_{3}\left(\omega^{3}+\omega^{-1}\right)\|f\|_{0, \Omega},
\end{aligned}
$$

Proof. First notice that the inequality (3.13) still holds in the two-dimensional case. Then by (3.13) and (4.5) we get

$$
\begin{aligned}
& \|u\|_{0, \Omega}^{2} \leq\left\{\begin{array}{l}
C\left(1+|\ln \omega|^{2}\right)\|f\|_{0, \Omega}^{2} \\
\quad+C\left(1+\omega^{2}|\ln \omega|^{2}\right)\left(\varepsilon\|u\|_{0, \Omega}^{2}+\frac{1}{4 \varepsilon \omega^{2}}\|f\|_{0, \Omega}^{2}\right), \quad \text { if } \omega<r_{0} / \sqrt{2}, \\
C\|f\|_{0, \Omega}^{2}
\end{array}\right. \\
& +C\left(1+\omega^{2}\right)\left(\frac{\varepsilon}{\omega^{2}}\|u\|_{0, \Omega}^{2}+\frac{1}{4 \varepsilon}\|f\|_{0, \Omega}^{2}\right), \\
& \text { if } \omega \geq r_{0} / \sqrt{2} \text {. }
\end{aligned}
$$

Obviously, for the large frequency $\omega$ the above inequality is same as one in the three-dimensional case. So the final estimate is same too. For the small frequency $\omega$ there are two new factors in the right-hand side of the above 
inequality, one is $|\omega \ln \omega|^{2}$ and the other is $|\ln \omega|^{2}$. But $|\omega \ln \omega|$ is small for small $\omega$ and $|\ln \omega|$ is dominated by $\left|\frac{1}{\omega}\right|$ for small $\omega$, hence the desired estimate is also obtained for small frequency $\omega$.

\section{REMARKS}

The similar estimate for the equations of the motion for elastic and nearly elastic solids in the frequency is proved by one of us (see [6]). The analysis can also be carried out for the general elliptic problem with fairly reasonable boundary conditions in a domain with piecewise flat boundaries. An essential step is to obtain estimates similar to (3.5) and (4.5). The condition (1.3) is not necessary for the elliptic regularity estimates of Sections 3 and 4.

\section{ACKNOWLEDGMENT}

The authors would like to thank Professor Jim Douglas, Jr., for his several helpful suggestions. The authors also would like to thank the referee for the helpful comments and for bringing the following paper to their attention: $\mathbf{R}$. Brown and Z. Shen, The initial Dirichlet problem for a fourth-order parabolic equation in Lipschitz domain, Indiana University Math J. 39 (1990), which contains some results on existence and estimates of solutions for the Helmhotz equation.

\section{REFERENCES}

1. R. A. Adams, Sobolev Spaces, Academic Press, New York, NY, 1975.

2. A. P. Calderón, Cauchy integrals on Lipschitz curves and related operators, Proc. Nat. Acad. Sci. U.S.A. 74 (1977), 1324-1327.

3. R. R. Coifman, A. McIntosh and Y. Meyer, L'intégrale de Cauchy définit un opérateur borne sur $L^{2}$ pour les courbes lipschitzinennes, Ann. of Math. (2) 116 (1982), 361-387.

4. J. Douglas, Jr., J. E. Santos, and D. Sheen, Approximation of scalar waves in the spacefrequency domain, preprint.

5. J. Douglas, Jr., J. E. Santos, D. Sheen, and L. Schreyer, Frequency domain treatment of one-dimensional scalar waves, Math. Models Methods Appl. Sci. 3 (1993), 171-194.

6. X. Feng, An elliptic regularity coefficient estimate for the equations of the motion for nearly elastic solids in the frequency domain, Comp. Appl. Math. (accepted).

7. P. Grisvard, Singularities in boundary value problems, Research Notes in Applied Mathematics, Vol. 22, Masson and Springer-Verlag, 1992.

8. C. L. Ravazzoli, J. Douglas, Jr., J. E. Santos, and D. Sheen, On the solution of the equations of motion for nearly elastic solids in the frequency domain, preprint.

9. C. J. Tarnter, Bessel functions with some applications, Hart, New York, 1969.

10. G. C. Verchota, Layer potential and regularity for the Dirichlet problem for Laplace's equation in Lipschitz domains, J. Funct. Anal. 59 (1984), 572-611.

11. G. N. Watson, A treatise on the theory of Bessel functions, 2nd ed., Cambridge Univ. Press, 1966.

Department of Mathematics, Wayne State University, Detroit, Michigan 48202

Current address: Department of Mathematics, The University of Tennessee, Knoxville, Tennesee 37996

E-mail address: xfeng@math.utk.edu

Department of Mathematics, Purdue University, West Lafayette, IN 47907

Current address: Department of Mathematics, Seoul National University, Seoul 151-742, Korea

E-mail address: sheen@math.snu.ac.kr 